Pathologe 2010 $31: 5$

DOI 10.1007/s00292-009-1238-9

Online publiziert: 15. Januar 2010

(c) Springer-Verlag 2010

\author{
M.L. Hansmann \\ Senckenbergisches Institut für Pathologie, Klinikum der Johann-Wolfgang-Goethe- \\ Universität Frankfurt
}

\title{
Aktuelle Tumorpathologie - Relevanz für die Klinik
}

Das vorliegende Heft von „Der Pathologe" und das IAP-Symposium 2010 haben "Aktuelle Tumorpathologie - Relevanz für die Klinik" zum Thema. Der Zeitpunkt erscheint gegeben, sich mit aktuellen Entwicklungen und Schwerpunkten in der modernen pathologisch-anatomischen Tumordiagnostik auseinanderzusetzen, da viele neue Entwicklungen stattgefunden haben oder gerade anlaufen. Noch vor wenigen Jahren klaffte, von einigen Ausnahmen abgesehen, eine breite Lücke zwischen konventioneller morphologischer und moleklarer Tumordiagnostik. Die Morphologie war vor allen Dingen relevant für die tägliche Diagnostik, während die Molekularpathologie eher ein Werkzeug der Forschung darstellte und nur begrenzten Praxisbezug zeigte.

Zurzeit befinden wir uns allerdings in der schon etwas überraschenden Situation, dass die molekularpathologische Diagnostik häufig nicht nur ergänzend zur konventionellen Diagnostik zu sehen ist, sondern dass sie ein Muss für viele Therapieentscheidungen darstellt. Beispielhaft seien die Mutationsanalysen für Rezeptoren wie c-Kit, RAS, RAF, EGFR u. a. genannt, die nach Mikrodissektion des Gewebes durch den Pathologen durchgeführt werden und dem Patienten ganz neue Therapieoptionen ermöglichen. Die auf den einzelnen Patienten zugeschnittene Therapie wird mehr und mehr Wirklichkeit und ist Ausdruck und Folge moderner Tumorpathologie.

Diese individualisierte Vorgehensweise wurde erst durch den Nachweis und die nähere molekulare Definition der therapeutischen Targets im Tumorgewebe durch den Pathologen möglich.
Die Pathologie findet sich somit auf einem innovativen, rationalen und zudem, wenn man die Kosten der Therapien berücksichtigt, auf einem kostengünstigen Weg, der Diagnostik und Therapieoptionen verknüpft. Eine, wie ich meine, zukunftsweisende Kombination. Die moderne Tumorpathologie hat heutige diagnostische Herausforderungen erfolgreich angenommen und wir stellen uns, wie in den vorliegenden Beiträgen angeschnitten, im Hinblick auf die molekularen diagnostischen Verfahren folgende Fragen: „Was muss sein? Was soll sein? Was kann sein?" und „Was wird sein?".

Einige Antworten auf diese Fragen werden in den verschiedenen Artikeln dieses Heftes gegeben. Die Beiträge beschäftigen sich mit unterschiedlichen Gebieten der Diagnostik von Tumoren verschiedener Organsysteme, wie Gastrointestinaltrakt, Lunge, Knochenmark, Lymphknoten, Niere und Mamma.

Die moderne Pathologie hat nicht nur eine, sondern viele Türen aufgestoßen und ist dabei zu vermitteln, dass sie ein spannendes und aufregendes diagnostisches Fach ist und auch in Zukunft bleiben wird.

h. L. Xan monm

Prof. Dr. Dr. h.c. M.-L. Hansmann

\section{Korrespondenzadresse}

Prof. Dr. Dr. h.c. M.L. Hansmann

Senckenbergisches Institut für Pathologie, Klinikum der Johann-Wolfgang-

Goethe-Universität Frankfurt

Theodor-Stern-Kai 7, 60590 Frankfurt

m.l.hansmann@em.uni-frankfurt.de 